\title{
Disparities and survival in newly diagnosed gastric cancer in Hispanic patients in the United States: a propensity score matched analysis
}

\author{
Joshua Tseng^, James P. Miller, Jeffrey Johnson, Kevin Waters, Alexandra Gangi, Jun Gong, \\ Miguel Burch
}

Department of Surgery, Cedars-Sinai Medical Center, Los Angeles, CA, USA

Contributions: (I) Conception and design: J Tseng, JP Miller, J Johnson, M Burch; (II) Administrative support: J Tseng, A Gangi, J Gong, M Burch; (III) Provision of study materials or patients: J Tseng, JP Miller, J Johnson, M Burch; (IV) Collection and assembly of data: J Tseng, JP Miller, J Johnson; (V) Data analysis and interpretation: J Tseng, J Johnson, K Waters, A Gangi, J Gong, M Burch; (VI) Manuscript writing: All authors; (VII) Final approval of manuscript: All authors.

Correspondence to: Miguel Burch, MD. Department of Surgery, Cedars-Sinai Medical Center, 8635 W 3rd Street \#795W, Los Angeles, CA 90048, USA. Email: Miguel.burch@cshs.org.

Background: The burden of gastric cancer involving Hispanic patients in the United States is growing as both the population and the incidence of gastric cancer in this group increases. This burden is compounded by presentation with advanced disease and socioeconomic challenges shaping cancer care. We sought to describe the demographics, socioeconomic factors, treatment, and survival experience of Hispanic patients with gastric adenocarcinoma.

Methods: Patients with gastric adenocarcinoma diagnosed between 2004 and 2015 ( $\mathrm{n}=90,737)$ in the National Cancer Database were retrospectively identified. Patients of Hispanic ethnicity were compared against non-Hispanic white patients. Surgical cohort was further analyzed, and 1:1 propensity score matching was used to balance covariates between Hispanic and non-Hispanic white surgical patients. Survival was compared using Kaplan-Meier method. Cox regression was used to determine prognostic factors for survival. Results: Compared to non-Hispanic white patients, Hispanic patients are more likely to be younger, female, and healthier. They were more likely to be uninsured, reside in poorer neighborhoods and reside in areas with lower rates of education. Hispanic patients were more likely to live in a metropolitan area, travel shorter distances for healthcare, and receive treatment at an academic and high volume centers. Hispanic patients were more likely to have higher stage disease presentation, higher grade tumors, lymphovascular invasion, and poorly cohesive adenocarcinoma. Hispanic patients were more likely to receive surgery, but less likely to receive adjuvant therapy. In Cox regression of all patients, unmatched surgical patients, and matched surgical patients, Hispanic ethnicity was an independent prognostic factor of improved survival.

Conclusions: Hispanic patients with gastric adenocarcinoma present with several unfavorable clinicopathologic and socioeconomic factors. Paradoxically, these patients demonstrate improved survival. Further study is warranted to characterize disease biology in this population.

Keywords: Gastric cancer; The National Cancer Database (NCDB); ethnicity; Hispanic; propensity score matching

Submitted Apr 14, 2021. Accepted for publication Jun 30, 2021.

doi: 10.21037/jgo-21-207

View this article at: https://dx.doi.org/10.21037/jgo-21-207

\footnotetext{
^ ORCID: 0000-0002-9112-8792.
} 


\section{Introduction}

Although the incidence and mortality of gastric cancer has declined over the past few decades, it is still the $5^{\text {th }}$ most common cancer and the $3^{\text {rd }}$ most frequent cause of cancerrelated deaths worldwide $(1,2)$.

The prognostic significance of race and ethnicity in gastric cancer is unclear. Gastric cancer patients in Asia have consistently demonstrated a survival benefit compared to patients in the West (3-6). Some authors postulate that these findings stem from differences in screening guidelines and treatment regimen between Eastern and Western countries (7-13). However, Asian patients with gastric cancer in the United States still retain this survival benefit, which questions the hypothesis that regional variations in treatment alone account for these differences (14-16). Instead, differences in tumor biology may significantly contribute to these racial disparities.

While the incidence of gastric cancer has decreased for the general population, the burden of gastric cancer involving Hispanic patients in the United States has paradoxically grown (17). This burden is compounded by their tendency to present with advanced, aggressive disease, as well as socioeconomic challenges that typically prevent access to cancer care. For these reasons, we sought to describe the patient demographics, socioeconomic factors, tumor biology, and management of Hispanic patients with gastric cancer, and the impact of these factors on survival.

We present the following article in accordance with the STROBE reporting checklist (available at https://dx.doi. org/10.21037/jgo-21-207).

\section{Methods}

The National Cancer Database (NCDB) is a clinical oncology database sponsored by the American College of Surgeons and the American Cancer Society. It captures data from more than 1,500 Commission on Cancer (CoC)accredited facilities and represents over 70 percent of newly diagnosed cancer cases nationwide. These facilities are categorized as "Community Cancer Program" (CCP), "Comprehensive Community Cancer Program" (CCCP), "Academic/Research Program" (Academic), and Integrated Network Cancer Program" (INCP) by the CoC.

The NCDB was retrospectively queried for all patients of Hispanic and non-Hispanic white ethnicity from 2006-2015 with a diagnosis of gastric adenocarcinoma based on the 2010 WHO classification of digestive tumors (18). ICD-0-
3 histology codes for gastric adenocarcinoma for this study included poorly cohesive/signet ring cell carcinoma (8142, $8145,8490)$ and other $(8140,8144,8210,8211,8255,8260$, $8480-8481,8560,8574)$. Race and ethnicity were assessed using the same 1997 Office of Management and Budget standards as the US Census Bureau, in which white race includes a person having origins in any of the original peoples of Europe, the Middle East, or North Africa. Only patients with one primary cancer were included (NAACCR \#560, code 00 or 01 ). Patients who were not treated at the reporting facility were excluded from the study (NAACCR $\# 610$, code 00$)$. For patients who were staged by AJCC $6^{\text {th }}$ and $7^{\text {th }}$ edition, collaborative stage site-specific factor 25 (Schema Discriminator: EsophagusGEJunction (EGJ)/ Stomach) records the distance of the tumor midpoints from the EGJ and was used to exclude Siewart I-III tumors from the study. Patients with missing information of the preceding variables were excluded.

The patient variables collected included age, gender, Charlson-Deyo Combined Comorbidity score (CDCC), insurance status, income quartile, education quartile, population density (metropolitan, urban, and rural), distance traveled to receive care, and the facility type where the patient received their care. Distance traveled was categorized into $<10,10-25$, and $>25$ miles. Age was categorized into $<40,40-70$, and $>70$ years old. The gastric cancer case volume of each unique facility was quantified and categorized into $<50^{\text {th }}, 50-75^{\text {th }}$, and $>75^{\text {th }}$ percentile, corresponding to $<56,57-109$, and $>109$ cases over 11 years, respectively. $>75^{\text {th }}$ percentile centers were described as "high-volume centers" in this study.

Tumor characteristics assessed include clinical and pathologic stage, tumor grade, presence of lymphovascular invasion, and histologic subtype (poorly cohesive/signet and other). Data on tumor location was obtained using ICD-O-3 topography codes, classifying cardia/fundus as "proximal", and body/antrum/pylorus as "distal". Tumors of the lesser curvature, greater curvature, overlapping lesions, and stomach NOS were classified as "other".

Treatment patterns (surgery, chemotherapy, and radiation) were described for each analytic stage, which is a combination of pathologic staging when available, and clinical staging if pathologic staging is not available. For patients undergoing surgery, margin status, perioperative chemotherapy, perioperative radiation therapy, and number of lymph nodes (LN) retrieved were also assessed. As the National Comprehensive Cancer Network (NCCN) recommends performing a lymphadenectomy with a 
minimum of $16 \mathrm{LN}$ harvested, we assessed the proportion of patients who underwent lymphadenectomy with 16 or more LN retrieved (19). Patients were categorized by their ethnicity for comparison. Patients with analytic stage I-IV gastric cancer who underwent surgery were further analyzed before and after propensity score matching.

No preregistration exists for the reported studies reported in this article. The study was conducted in accordance with the Declaration of Helsinki (as revised in 2013), and was determined to be exempt from Institutional Review Board (IRB) oversight due to the de-identified nature of the data. The data is available upon request from the authors.

\section{Statistical analysis}

Categorical variables were listed in absolute numbers and percent prevalence (\%) in the study group. The MannWhitney $\mathrm{U}$ test was used to compare the medians of nonparametric continuous variables for significance. Categorical variables were compared using the Pearson's chi-squared test. Kaplan-Meier survival curves for overall survival for each individual pathologic stage were compared by the log-rank test. A multivariable Cox proportionalhazards regression model was used to identify independent prognostic factors for survival. Lymphovascular invasion and Grade were not used in the Cox regression model due to their high rates of missing data. For the surgical cohort, patient and tumor characteristics were compared. Subsequently, 1:1 propensity score matching of Hispanic to non-Hispanic white surgical patients was performed using logistic regression analysis with nearest-neighbor matching with a caliper of 0.001 . Covariate balance was assessed with the Standardized Mean Difference (SMD), in which a SMD $<0.1$ represents balanced covariates between the two groups (20). Statistical analyses were performed using IBM SPSS, Version 25.0 (SPSS, Inc, Armonk, NY, USA).

\section{Results}

A total of 90,737 patients with gastric adenocarcinoma of Hispanic and non-Hispanic white ethnicity were identified, of which 12,671 (14.0\%) were Hispanic and 78,066 (86.0\%) were non-Hispanic (Table 1). The number of new gastric cancer diagnoses in Hispanic patients increased from 867 to 1,208 cases (39.3\%) from 2004-2015, compared to 6,430 to $6,676(3.8 \%)$ in non-Hispanic white patients. The majority of patients were ages $41-70(53.9 \%)$ and ages $70+(42.3 \%)$, while $3.8 \%$ of patients were ages $18-40$. The majority of patients lived in metropolitan areas $(84.2 \%)$ and traveled $<10$ miles to receive care $(50.4 \%)$. The majority of patients were treated at CCCP's and academic programs $(40.6 \%$ and $39.6 \%$, respectively). A total of 32,971 (41.0\%) patients had metastatic disease, and 19,787 (21.8\%) had poorly cohesive/ signet ring cell type adenocarcinoma. The median followup was 27.3 months (IQR, 12.4-52.8 months).

Compared to non-Hispanic patients, Hispanic patients were younger $(61$ vs. 68 years old, $\mathrm{P}<0.01)$ and healthier (CDCC $0,72.7 \%$ vs. $69.0 \%, \mathrm{P}<0.01$ ) (Table 1). Hispanic patients were more likely to live in the poorest neighborhoods $(27.2 \%$ vs. $14.4 \%, \mathrm{P}<0.01)$ and areas with the lowest rates of education $(53.1 \%$ vs. $13.8 \%, \mathrm{P}<0.01)$. The majority of Hispanic patients lived in metropolitan areas $(94.9 \%)$ and traveled $<10$ miles to receive their care (64.4\%). They were also more likely to receive care at an academic program $(44.2 \%$ vs. $38.9 \%, \mathrm{P}<0.01)$ and programs with the highest case volumes $(70.9 \%$ vs. $57.2 \%, \mathrm{P}<0.01)$. Hispanic patients were more likely to have metastatic disease ( $47.7 \%$ vs. $39.9 \%, \mathrm{P}<0.01)$, poorly cohesive/signet ring cell type adenocarcinoma $(32.8 \%$ vs. $20.0 \% \mathrm{P}<0.01)$, and tumors located in the distal stomach (34.9\% vs. $20.2 \%$ $\mathrm{P}<0.01)$.

Table 2 describes the rates of treatment by analytic stage. Hispanic patients were more likely to receive surgery for each individual stage from 1-4, but were also less likely to receive radiation therapy for stages $0-4$, and less likely to receive chemotherapy for stages $2-3$.

Kaplan-Meier survival curves of Hispanic vs. nonHispanic patients by analytic stage are described in Figure $1 A-D$. For each analytic stage, Hispanic patients had a longer median survival time compared to nonHispanic patients by the log rank test (stage $1,106.2 \mathrm{vs}$. 64.9 months, $\mathrm{P}<0.01$; stage 2, 51.9 vs. 29.2 months, $\mathrm{P}<0.01$; stage $3,23.0$ vs. 17.2 months, $\mathrm{P}<0.01$; stage $4,7.1$ vs. 5.8 months, $\mathrm{P}<0.01)$.

A Cox proportional-hazards regression model was used to identify independent prognostic factors for overall survival of the entire cohort (Table 3). Factors associated with worse survival include age $>70$ (HR 1.28, 95\% CI: 1.25-1.31), higher CDCC score (CDCC 3, HR 1.53, 95\% CI: 1.45-1.62), pathologic stage (stage 2, HR 1.60, 95\% CI: 1.39-1.83; stage 3, HR 2.56, 95\% CI: 2.23-2.93; stage 4, HR 4.03, 95\% CI: 3.52-4.61), and poorly cohesive/ signet ring cell type adenocarcinoma (HR 1.22, $95 \%$ CI: 1.19-1.24). Factors associated with improved survival include Hispanic ethnicity (HR 0.76, 95\% CI: 0.73-0.78), 
Table 1 Patient and tumor characteristics of entire cohort

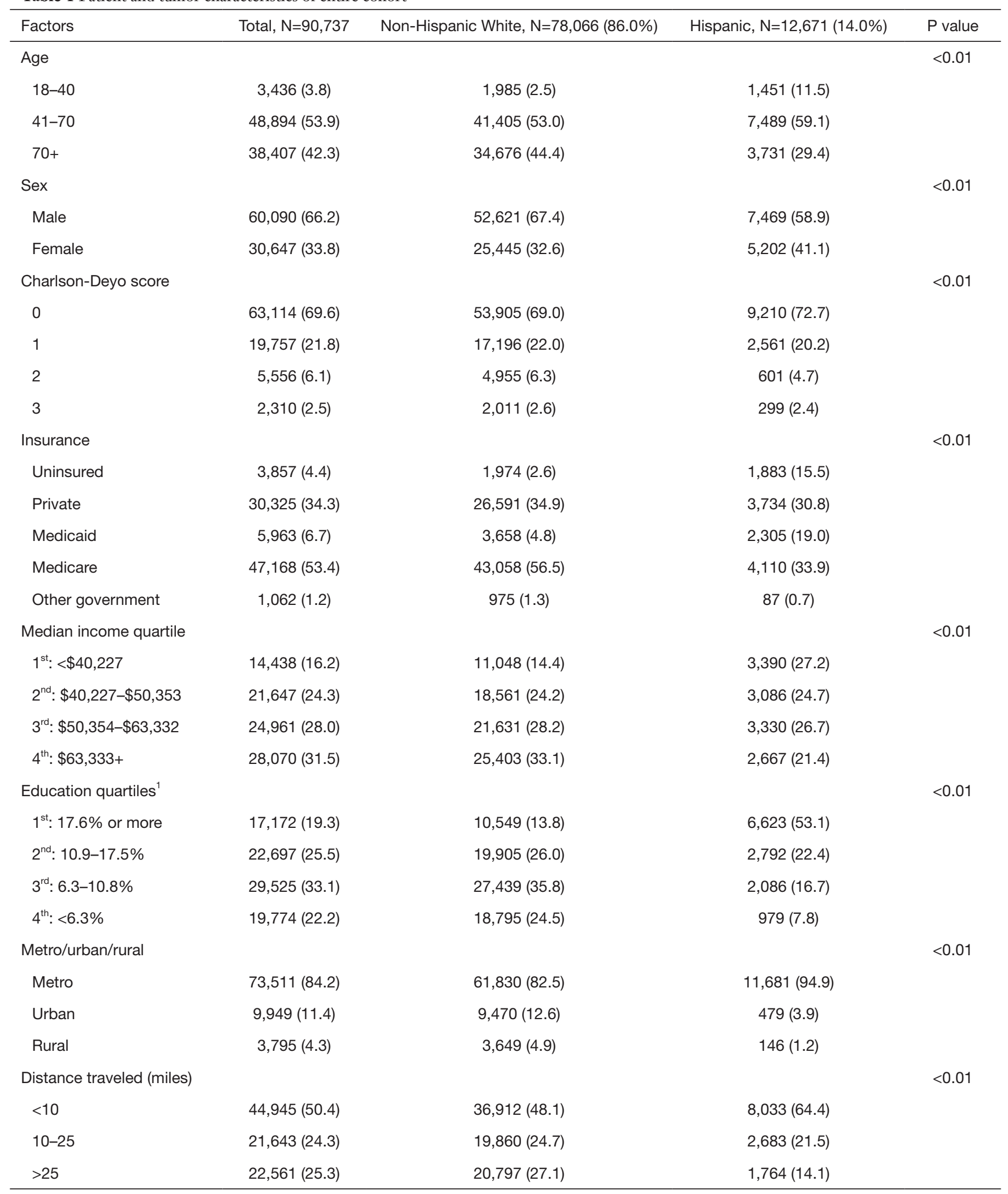

Table 1 (continued) 
Table 1 (continued)

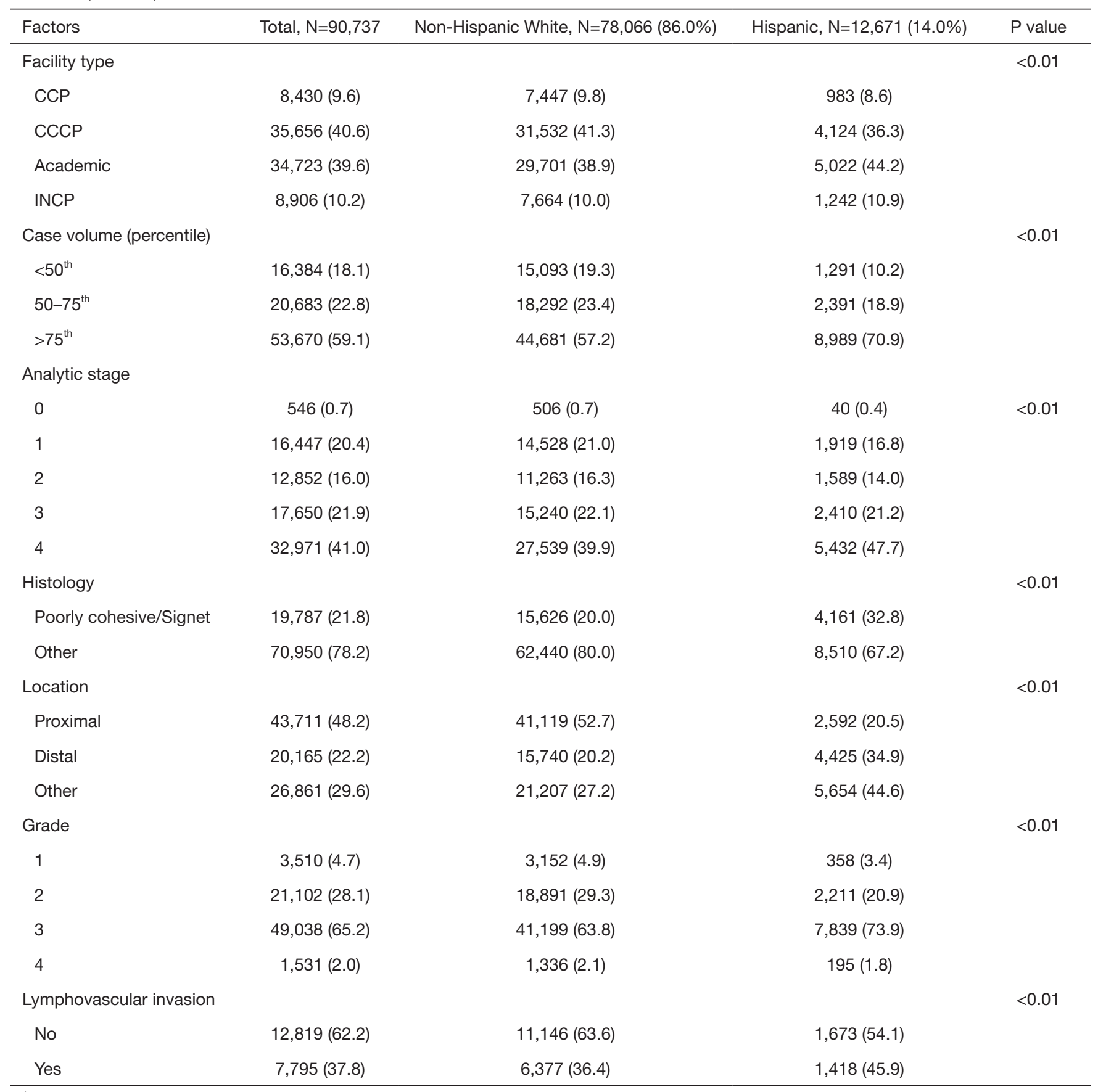

${ }^{1}$, percentage of population with no high school degree. CCP, Community Cancer Program; CCCP, Comprehensive Community Cancer Program; INCP, Integrated Network Cancer Program.

higher income quartiles (quartile 4, HR 0.84, 95\% CI: 0.81-0.87), private insurance (HR 0.91, 95\% CI: 0.87-0.96), receiving care at an academic program (HR 0.89, 95\% CI: 0.85-0.93), receiving care at a high volume center (HR 0.90,
95\% CI: 0.87-0.92), and receiving surgery, chemotherapy, or radiation (HR 0.42, 95\% CI: 0.41-0.43; HR 0.56, 95\% CI: 0.55-0.57; HR 0.97, 95\% CI: 0.95-0.99), respectively. There was no statistically significant difference between 
Table 2 Treatment by analytic stage

\begin{tabular}{|c|c|c|c|c|c|c|}
\hline Stage & Treatment & Subgroup & Total, N=90,737 & Non-Hispanic White, N=78,066 (86.0\%) & Hispanic, $\mathrm{N}=12,671$ (14.0\%) & $P$ value \\
\hline \multirow{4}{*}{0} & & Yes & $143(77.1)$ & $381(76.8)$ & $32(80.0)$ & \\
\hline & Radiation & No & $321(58.8)$ & $291(57.5)$ & $30(75.0)$ & 0.031 \\
\hline & & Yes & $225(41.2)$ & $215(42.5)$ & $10(25.0)$ & \\
\hline & & Yes & $292(53.5)$ & $274(54.2)$ & $18(45.0)$ & \\
\hline \multirow[t]{4}{*}{1} & Surgery & No & $3,594(22.1)$ & $3,212(22.3)$ & $382(20.1)$ & 0.025 \\
\hline & & Yes & $12,696(77.9)$ & $11,174(77.7)$ & $1,522(79.9)$ & \\
\hline & Radiation & No & $13,043(79.3)$ & $11,393(78.4)$ & $1,650(86.0)$ & $<0.01$ \\
\hline & & Yes & $4,305(26.2)$ & $3,779(26.0)$ & $526(27.4)$ & \\
\hline \multirow[t]{6}{*}{2} & Surgery & No & 3,196 (25.2) & 2,891 (26.0) & 305 (19.3) & $<0.01$ \\
\hline & & Yes & $9,500(74.8)$ & $8,227(74.0)$ & $1,273(80.7)$ & \\
\hline & Radiation & No & $6,358(49.5)$ & 5,377 (47.7) & $981(61.7)$ & $<0.01$ \\
\hline & & Yes & $6,494(50.5)$ & $5,886(52.3)$ & $608(38.3)$ & \\
\hline & Chemotherapy & No & $4,602(35.8)$ & 3,991 (35.4) & $611(38.5)$ & 0.019 \\
\hline & & Yes & $8,250(64.2)$ & 7,272 (64.6) & $978(61.5)$ & \\
\hline 3 & Surgery & No & $4,782(27.4)$ & $4,290(28.5)$ & $492(20.6)$ & $<0.01$ \\
\hline \multirow[t]{6}{*}{4} & Surgery & No & $27,443(83.9)$ & 23,022 (84.3) & 4,421 (82.2) & $<0.01$ \\
\hline & & Yes & $5,256(16.1)$ & $4,296(15.7)$ & $960(17.8)$ & \\
\hline & Radiation & No & 26,671 (80.9) & $21,890(79.5)$ & $4,781(88.0)$ & $<0.01$ \\
\hline & & Yes & $6,300(19.1)$ & $5,649(20.5)$ & 651 (12.0) & \\
\hline & Chemotherapy & No & $14,295(43.4)$ & 11,895 (43.2) & $2,400(44.2)$ & 0.179 \\
\hline & & Yes & $18,676(56.6)$ & $15,644(56.8)$ & $3,032(55.8)$ & \\
\hline
\end{tabular}

tumors located proximally $v s$. distally (HR 0.99, 95\% CI: 0.98-1.02).

\section{Surgical cohort}

Patients who underwent surgery were further analyzed to assess for racial/ethnic disparities (Table 4). The majority of operations were performed at either an academic institution $(45.4 \%)$ or CCCP $(36.9 \%)$ and in high-volume centers (66.6\%). Furthermore, $46.3 \%$ underwent lymphadenectomy with $\geq 16 \mathrm{LN}$ recovered, and $16.5 \%$ had positive margins after surgery. And $43.4 \%$ did not receive chemotherapy, 
A

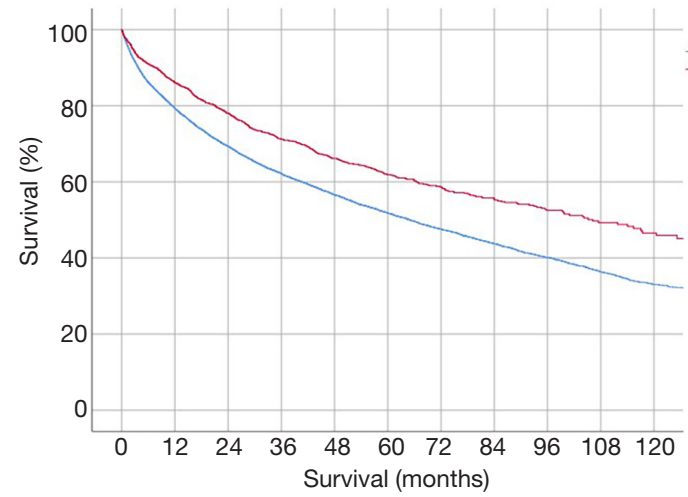

C

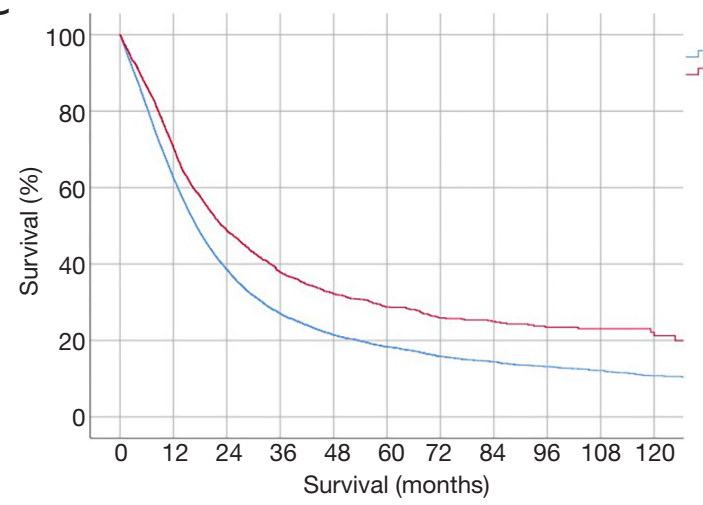

B

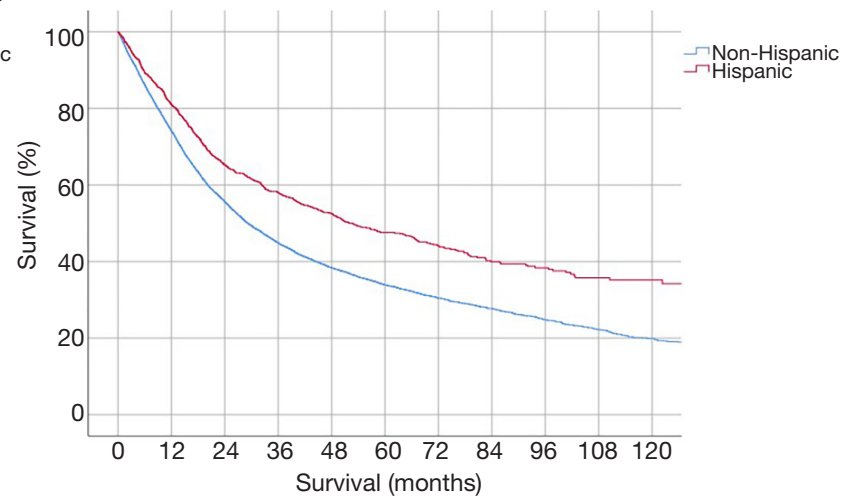

D

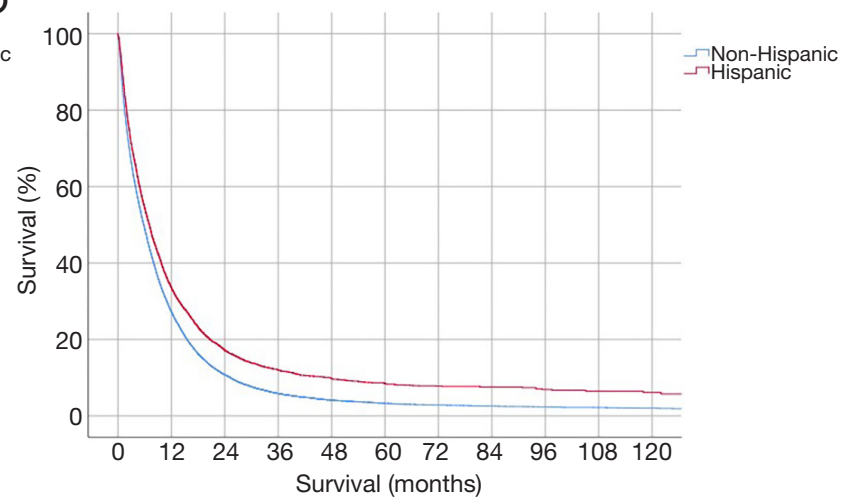

Figure 1 Survival of Hispanic $v s$. non-Hispanic White patients by analytic stage: (A) stage 1; (B) stage 2; (C) stage 3; (D) stage 4.

$6.0 \%$ received perioperative chemotherapy, $25.9 \%$ received neoadjuvant chemotherapy, and $24.8 \%$ received adjuvant chemotherapy.

Hispanic patients undergoing surgery were more likely to have unfavorable socioeconomic factors compared to nonHispanic patients, such as low income $(25.4 \% v s .14 .2 \%$ in $1^{\text {st }}$ quartile, $\left.\mathrm{P}<0.01\right)$, low education $\left(50.9 \%\right.$ vs. $13.3 \%$ in $1^{\text {st }}$ quartile, $\mathrm{P}<0.01)$, and uninsured status $(10.9 \%$ vs. $1.8 \%$, $\mathrm{P}<0.01)$. In terms of surgical quality, Hispanic patients were more likely to have positive margins after resection (19.5\% vs. $16.0 \%, \mathrm{P}<0.01)$, but were more likely to have $\geq 16 \mathrm{LN}$ retrieved $(54.7 \%$ vs. $44.0 \%, \mathrm{P}<0.01)$.

1:1 propensity score matching with nearest neighbor matching and a caliper of 0.001 was performed to balance the covariates present in Table 4. A total of 3,590 Hispanic and 3,590 non-Hispanic white patients were matched. All covariates had a standardized mean difference $<0.1$ after matching, demonstrating good balance between the two groups for each covariate.

A Cox proportional-hazards regression model was used to identify independent prognostic factors for overall survival of the surgical cohort (Table 5). Similar to the results from the entire cohort, factors associated with increased mortality include age $>70$ (HR 1.41, 95\% CI: 1.35-1.47), higher CDCC score (CDCC 3, HR 1.54, 95\% CI: 1.39-1.70), analytic stage (stage 2, HR 1.83, 95\% CI: 1.49-2.24; stage 3, HR 3.34, 95\% CI: 2.72-4.09; stage 4, HR 5.76, 95\% CI: 4.69-7.08), poorly cohesive adenocarcinoma (HR 1.14, 95\% CI: 1.09-1.19), and positive margins (HR 1.79, 95\% CI: $1.72-1.86)$. Factors associated with improved survival include Hispanic ethnicity (HR 0.78, 95\% CI: 0.73-0.82), higher median income (quartile 4, HR 0.89, $95 \%$ CI: $0.83-0.95$ ), receiving care at an academic program (HR 0.86, 95\% CI: 0.79-0.93), and receiving care at a high-volume center (HR 0.89, 95\% CI: 0.84-0.94). Lymphadenectomy with $\geq 16 \mathrm{LN}$ was associated with a survival benefit (HR 0.84 , 95\% CI: 0.81-0.87), as well as neoadjuvant chemotherapy (HR 0.75, 95\% CI: 0.71-0.79), adjuvant chemotherapy (HR $0.63,95 \%$ CI: $0.60-0.67$ ), perioperative chemotherapy (HR 0.64, 95\% CI: 0.59-0.70), and adjuvant radiation (HR 0.86, 95\% CI: 0.82-0.91).

A Cox proportional-hazards regression was performed on 
Table 3 Cox proportional-hazards regression of overall survival for entire cohort

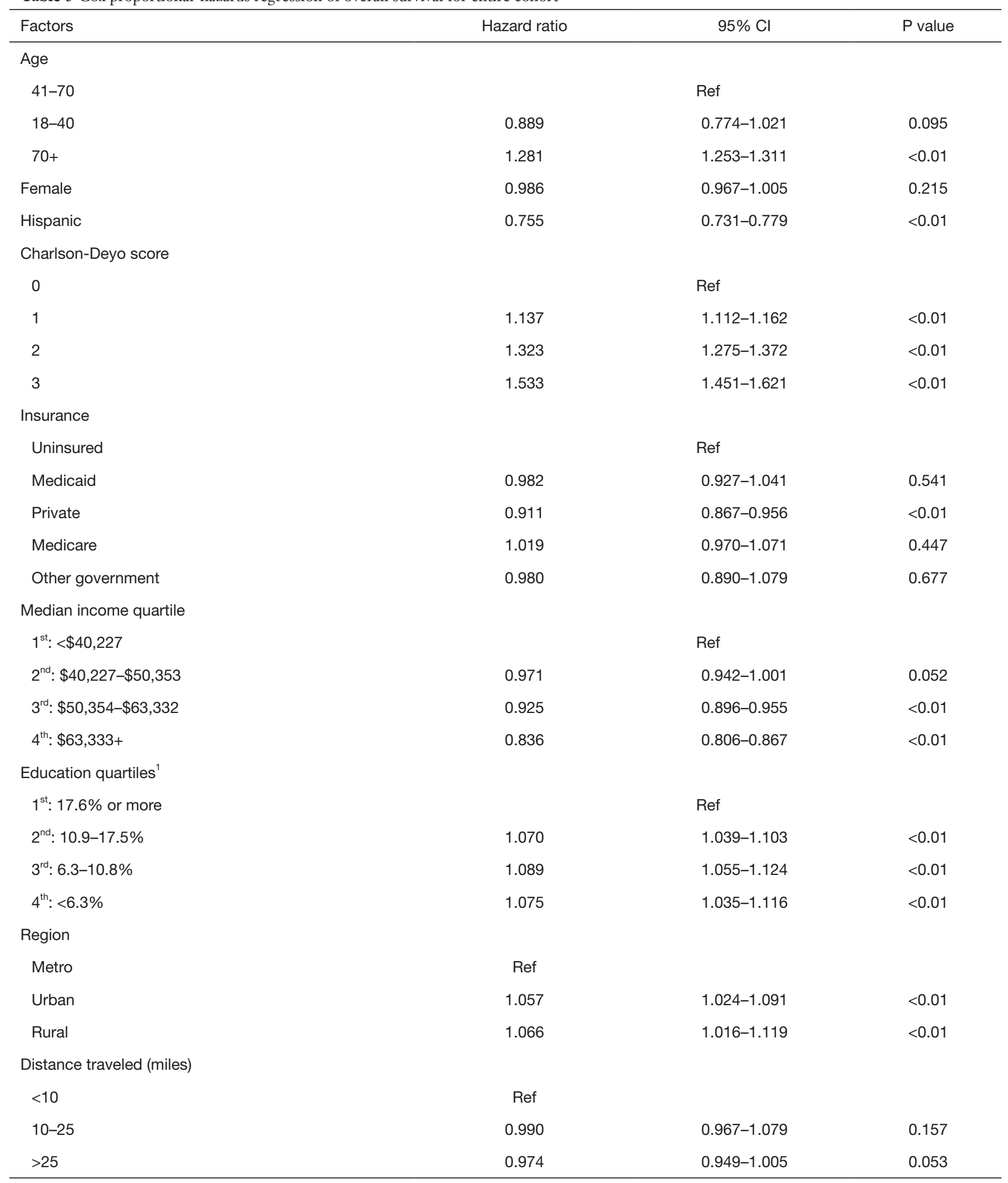

Table 3 (continued) 
Table 3 (continued)

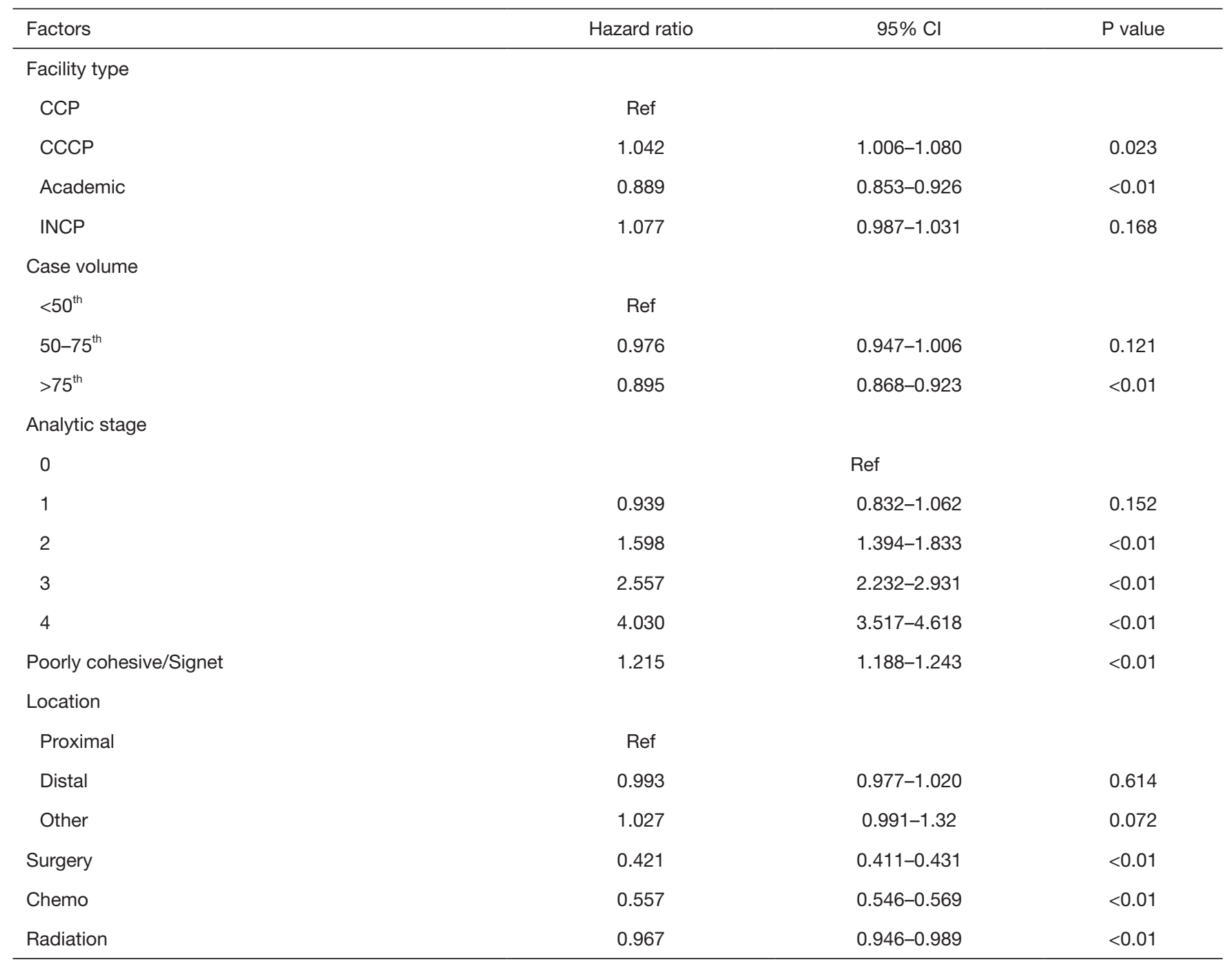

${ }^{1}$, percentage of population with no high school degree. CCP, Community Cancer Program; CCCP, Comprehensive Community Cancer Program; INCP, Integrated Network Cancer Program.

the propensity score matched surgical cohort (Table 5). Similar to the results for the unmatched surgical cohort, factors associated with increased mortality include age $>70$ (HR 1.46, 95\% CI: 1.35-1.58), higher CDCC score (CDCC 3, HR 1.61, 95\% CI: 1.45-1.77), higher stage (stage 2, HR 2.92, 95\% CI: 1.51-5.65; stage 3, HR 5.85, 95\% CI: 3.03-11.29; stage 4, HR 9.43, 95\% CI: 4.87-18.23), poorly cohesive histology (HR 1.29, 95\% CI: $1.20-1.39$ ), and positive margins (HR 1.82, 95\% CI: $1.68-1.97)$. Distance traveled $>25$ miles for care was associated with higher hazard (HR 1.15, 95\% CI: 1.04-1.28). Factors associated with improved survival include Hispanic ethnicity (HR 0.80, 95\% CI: 0.75-0.85), higher income quartile ( $4^{\text {th }}$ quartile, HR $0.78,95 \%$ CI: $\left.0.69-0.89\right)$, academic program (HR 0.90, 95\% CI: 0.82-0.96), higher case volume center (HR 0.84, 95\% CI: 0.73-0.95), lymphadenectomy $\geq 16$ LN (HR 0.80, 95\% CI: 0.75-0.86), neoadjuvant chemotherapy (HR 0.78, 95\% CI: 0.70-0.87), adjuvant chemotherapy (HR $0.62,95 \%$ CI: $0.57-0.68$ ), perioperative chemotherapy (HR $0.61,95 \%$ CI: $0.52-0.71$ ), and adjuvant radiation therapy (HR 0.82, 95\% CI: 0.74-0.90).

\section{Discussion}

Although the prognostic significance of ethnicity and 


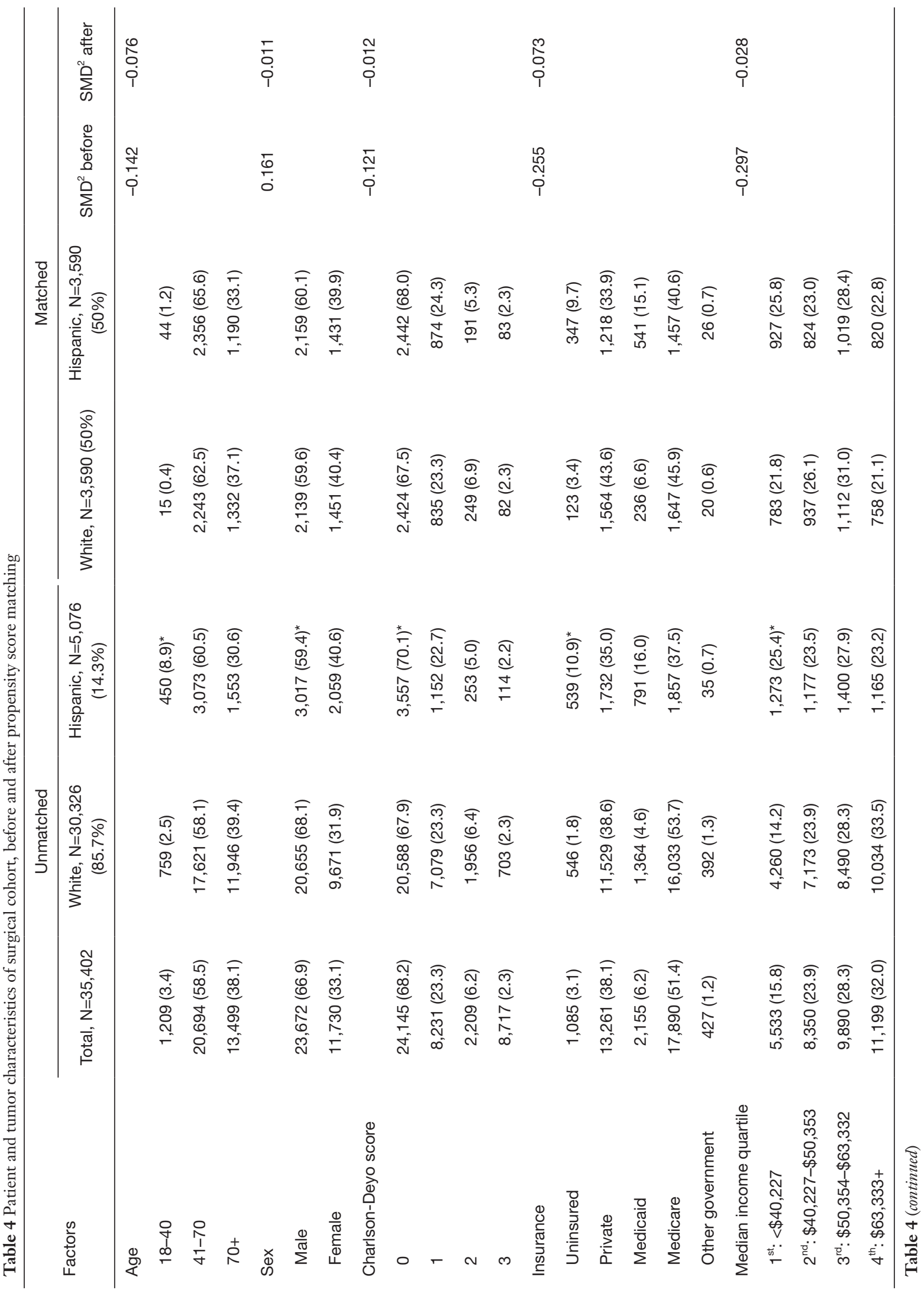




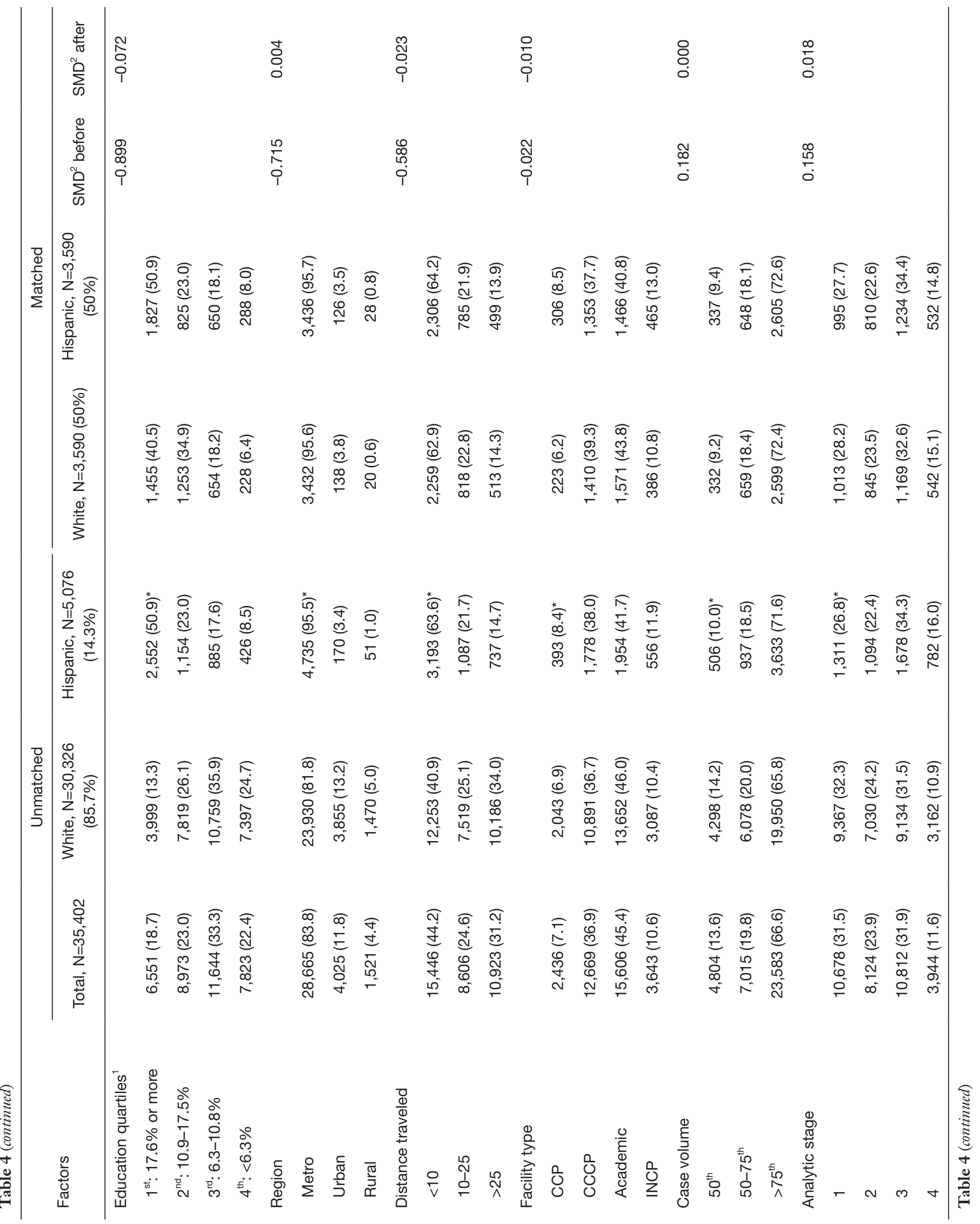




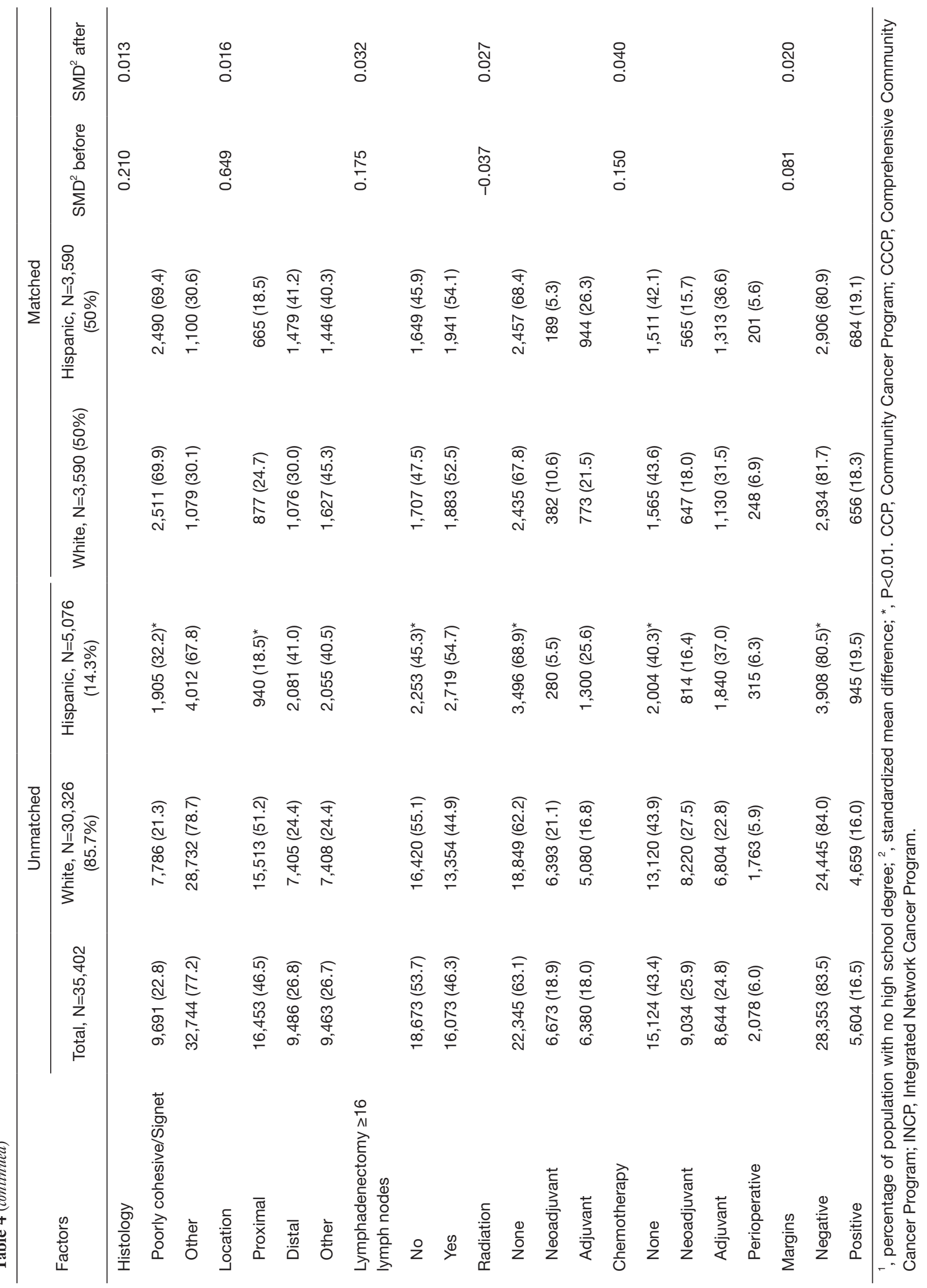


Table 5 Cox proportional-hazards regression for overall survival of surgical cohort, before and after propensity score matching

\begin{tabular}{|c|c|c|c|c|}
\hline Factors & \multicolumn{2}{|l|}{ Unmatched } & \multicolumn{2}{|l|}{ Matched } \\
\hline \multicolumn{5}{|l|}{ Age } \\
\hline $41-70$ & Ref & & Ref & \\
\hline $18-40$ & $0.865(0.668-1.120)$ & 0.272 & $0.712(0.462-1.095)$ & 0.122 \\
\hline Female & $0.938(0.905-0.972)$ & $<0.01$ & $0.970(0.908-1.037)$ & 0.377 \\
\hline \multicolumn{5}{|l|}{ Race } \\
\hline White & Ref & & Ref & \\
\hline Hispanic & $0.775(0.733-0.819)$ & $<0.01$ & $0.799(0.748-0.854)$ & $<0.01$ \\
\hline 1 & $1.113(1.072-1.156)$ & $<0.01$ & $1.132(1.090-1.175)$ & $<0.01$ \\
\hline 2 & $1.287(1.209-1.370)$ & $<0.01$ & $1.320(1.241-1.404)$ & $<0.01$ \\
\hline 3 & $1.536(1.389-1.698)$ & $<0.01$ & 1.606 (1.454-1.773) & $<0.01$ \\
\hline \multicolumn{5}{|l|}{ Median income quartile } \\
\hline $1^{\text {st }}:<\$ 40,227$ & Ref & & Ref & \\
\hline $2^{\text {nd. }}: \$ 40,227-\$ 50,353$ & $0.979(0.927-1.033)$ & 0.437 & $1.013(0.923-1.111)$ & 0.790 \\
\hline 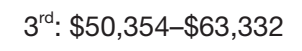 & $0.947(0.894-1.003)$ & 0.064 & $1.012(0.920-1.114)$ & 0.807 \\
\hline $4^{\text {th }}: \$ 63,333+$ & $0.891(0.834-0.952)$ & $<0.01$ & $0.784(0.693-0.886)$ & $<0.01$ \\
\hline \multicolumn{5}{|l|}{ Distance traveled } \\
\hline$<10$ miles & Ref & & Ref & \\
\hline 10-25 miles & $1.005(0.964-1.047)$ & 0.825 & $1.042(0.962-1.128)$ & 0.311 \\
\hline$>25$ miles & $0.999(0.953-1.048)$ & 0.975 & 1.149 (1.035-1.275) & $<0.01$ \\
\hline \multicolumn{5}{|l|}{ Region } \\
\hline Metro & Ref & & Ref & \\
\hline Urban & $1.054(0.996-1.115)$ & 0.070 & $0.970(0.811-1.159)$ & 0.735 \\
\hline Rural & $1.076(0.991-1.169)$ & 0.081 & 0.937 (0.634-1.386) & 0.745 \\
\hline \multicolumn{5}{|l|}{ Insurance } \\
\hline Uninsured & Ref & & Ref & \\
\hline Private & $0.955(0.861-1.060)$ & 0.385 & $1.032(0.895-1.191)$ & 0.661 \\
\hline
\end{tabular}

Table 5 (continued) 
Table 5 (continued)

\begin{tabular}{|c|c|c|c|c|}
\hline Factors & \multicolumn{2}{|l|}{ Unmatched } & \multicolumn{2}{|l|}{ Matched } \\
\hline Medicaid & $0.994(0.881-1.122)$ & 0.927 & $1.040(0.881-1.228)$ & 0.643 \\
\hline Medicare & $1.085(0.977-1.205)$ & 0.102 & $1.128(0.975-1.306)$ & 0.105 \\
\hline Other government & $1.065(0.885-1.281)$ & 0.507 & $0.850(0.525-1.378)$ & 0.511 \\
\hline \multicolumn{5}{|l|}{ Facility type } \\
\hline $\mathrm{CCP}$ & Ref & & Ref & \\
\hline CCCP & $0.996(0.927-1.070)$ & 0.912 & $1.102(0.956-1.270)$ & 0.182 \\
\hline Academic & $0.855(0.790-0.926)$ & $<0.01$ & $0.895(0.833-0.964)$ & $<0.01$ \\
\hline INCP & $0.960(0.883-1.044)$ & 0.335 & $0.941(0.798-1.110)$ & 0.471 \\
\hline $50-75^{\text {th }}$ & $0.984(0.927-1.045)$ & 0.597 & $0.847(0.740-0.968)$ & 0.015 \\
\hline$>75^{\text {th }}$ & $0.885(0.835-0.939)$ & $<0.01$ & $0.835(0.732-0.952)$ & $<0.01$ \\
\hline \multicolumn{5}{|l|}{ Analytic stage } \\
\hline 1 & Ref & & Ref & \\
\hline 2 & $1.829(1.492-2.241)$ & $<0.01$ & $2.922(1.512-5.646)$ & $<0.01$ \\
\hline 3 & 3.339 (2.726-4.089) & $<0.01$ & $5.848(3.031-11.286)$ & $<0.01$ \\
\hline 4 & $5.761(4.690-7.076)$ & $<0.01$ & $9.427(4.874-18.233)$ & $<0.01$ \\
\hline \multicolumn{5}{|l|}{ Location } \\
\hline Positive margins & $1.787(1.715-1.862)$ & $<0.01$ & $1.818(1.682-1.965)$ & $<0.01$ \\
\hline \multicolumn{5}{|l|}{ Chemo } \\
\hline None & Ref & & Ref & \\
\hline Neoadjuvant & $0.748(0.705-0.794)$ & $<0.01$ & $0.779(0.696-0.873)$ & $<0.01$ \\
\hline Adjuvant & $0.633(0.601-0.667)$ & $<0.01$ & $0.622(0.567-0.682)$ & $<0.01$ \\
\hline Perioperative & $0.642(0.593-0.695)$ & $<0.01$ & $0.610(0.524-0.711)$ & $<0.01$ \\
\hline \multicolumn{5}{|l|}{ Radiation } \\
\hline None & Ref & & Ref & \\
\hline Neoadjuvant & $1.033(0.969-1.101)$ & 0.314 & $1.308(0.895-1.204)$ & 0.620 \\
\hline Adjuvant & $0.863(0.818-0.910)$ & $<0.01$ & $0.816(0.742-0.898)$ & $<0.01$ \\
\hline
\end{tabular}


geographic location is frequently discussed in the gastric cancer literature, the experience of Hispanic patients is rarely mentioned. In our study, the burden of gastric cancer in the Hispanic population in the US significantly grew over the past decade compared to non-Hispanic white patients. Hispanic patients were also more likely to present with advanced, aggressive disease, and were more likely to be associated with disadvantageous socioeconomic factors such as lower income, lower education, and lack of insurance. Counterintuitively, Hispanic patients demonstrated improved survival compared to non-Hispanic white patients, even after adjusting for patient demographics, socioeconomic factors, tumor biology, and treatment regimen in both unmatched and matched cohorts. To our knowledge, this is the largest study that identifies Hispanic ethnicity as an advantageous prognostic factor for gastric cancer.

Many studies have identified racial disparities in gastric cancer outcomes. The majority of the data is focused on patients in Asia, as they consistently demonstrate improved survival compared to their Western counterparts (3-6). For example, 5-year survival rates in Japan (40-60\%) remain significantly higher than ones in the United States and Europe (15-20\%). These disparities are partly attributed to variations in diagnostic and treatment patterns between countries $(21,22)$. Some of the most notable practice differences in the East include early detection of gastric cancer through universal screening programs and surgical resection with systematic D2 node resections $(11,23,24)$. In contrast, the American Society for Gastrointestinal Endoscopy does not uniformly recommend endoscopic surveillance in the US due to the lack of studies to support this practice (25). Likewise, the American Gastoenterological Association suggests against routine use of endoscopic surveillance with the exception of certain high-risk populations such as racial/ethnic minorities (26). Furthermore, Western randomized trials support the use of perioperative chemotherapy (MAGIC and FLOT4) and adjuvant chemoradiotherapy (INT-0116), practices which have yet to be widely adopted in the East (5,10,23,27-29).

Although the management of gastric cancer differs between nations, the survival difference of Asian gastric cancer patients is still retained in Asians who reside in the US $(15,16)$. Many studies suggest that tumor biology may play a role, including factors such as tumor location (proximal vs. distal) and histology (poorly cohesive/signetring cell carcinoma) $(16,30)$.

Our study assessed the gastric cancer experience of over
12,000 Hispanic patients in the United States. The vast majority of Hispanic patients lived in metropolitan areas and traveled shorter distances to receive treatment at high volume centers. They were more likely to receive surgery, but less likely to receive adjuvant therapy. Hispanic patients were more likely to present with advanced, aggressive tumor characteristics, and were more likely to have unfavorable socioeconomic factors such as lower income, lower education rates, and lack of insurance. Hispanic patients who underwent surgery also had higher rates of positive margins. These factors were all associated with higher hazard for mortality on multivariate analysis.

Despite these negative factors, Hispanic patients had significantly improved overall survival compared to nonHispanic patients, stage for stage. These racial disparities remained significant on multivariate analysis after adjusting for patient characteristics, socioeconomic factors, tumor biology, and treatment patterns, and persisted after selecting for patients undergoing surgery and propensity score matching to balance differences in covariates. Overall, our findings suggest that differences in treatment patterns alone do not account for the racial disparities in gastric cancer. Further study is warranted to characterize disease biology in this population.

There is a paucity of studies assessing the experience of Hispanic patients with gastric cancer outside of the United States (31). Retrospective studies with small sample sizes describe a preponderance of distal tumors (75-100\%) and a variable rate of intestinal subtype $(35-100 \%)$. A recent abstract on gastric cancer in Mexico found a $120 \%$ increase in incidence of young gastric cancer patients (32). Furthermore, $70 \%$ of patients had poorly cohesive tumors and $76 \%$ were stage IV upon presentation, which was significantly higher (thus more aggressive) than the $32.8 \%$ of poorly cohesive tumors and $47.7 \%$ rate of metastatic disease in our study.

In the US, studies show that the incidence of gastric cancer has significantly increased over time in the Hispanic population in the United States. For example, a recent study of the SEER database showed that the incidence of gastric cancer rose from 1992 to 2011 for young Hispanic men and women -the only groups in the US to experience this growth (17). In addition, there was an increased incidence of stage 4 disease and poorly differentiated tumors for Hispanic patients. However, this study did not assess the prognostic effect of Hispanic race on survival. A small, single-center retrospective study of 20 Hispanic patients demonstrated no racial disparities in survival after 
adjusting for tumor characteristics, treatment, and patient demographics (33). Similarly, a single-center study of 301 Hispanic patients found that they were more likely to have poorly cohesive/signet histology, but again did not find a survival difference for Hispanic ethnicity (34).

A larger study of 3,466 Hispanic patients in Los Angeles County using its Cancer Surveillance Program from 19882000 demonstrated a survival benefit for Hispanic patients (HR 0.91, 95\% CI: 0.86-0.97), but no significant difference when looking at only those who underwent surgery (HR 0.94, 95\% CI: 0.85-1.05) (16). This study included Siewert type I and II tumors in the analysis and did not account for socioeconomic factors or neoadjuvant/perioperative chemotherapy.

Earlier studies demonstrated that cancers originating in the gastroesophageal junction and the cardia were associated with worse prognoses than those in the distal stomach $(35,36)$. However, these studies included Siewert type I and II tumors, which are now staged as esophageal cancers. Contemporary studies are mixed on the impact of tumor location survival $(37,38)$. In our study, we found no survival difference between patients with proximal tumors and those with distal tumors.

Our multivariate regression also identified academic programs and high-volume centers as prognostic factors for improved survival. This positive association between surgical volume and improved outcomes is corroborated by a recent study that examined the association between surgical hospital volume and outcomes using data obtained from the international CRITICS trial, and found significantly improved overall survival and diseasefree survival in higher-volume centers (39). These results emphasize the benefit of referring gastric cancer patients to tertiary centers with experience in gastric cancer.

\section{Limitations}

Our study has several limitations that are typical of large retrospective database studies. These include the potential for coding errors and missing data in the NCDB. As the NCDB only captures patients treated at Commission on Cancer-accredited programs, there may be significant selection bias in terms of race and ethnicity. In addition, both Hispanic and non-Hispanic white groups are highly heterogeneous, which may also introduce bias into our findings. In addition, our study is unable to account for confounding risk factors that have been traditionally associated with gastric cancer, such as diet, tobacco and alcohol use, obesity and the presence of $H$. pylori (40). Overall survival is the only survival information available in the database. Despite these limitations, the large sample size of the NCDB makes our study the largest study to date regarding racial and ethnic disparities in gastric cancer for people of Hispanic descent.

\section{Conclusions}

Hispanic patients with gastric adenocarcinoma present with several unfavorable clinicopathologic and socioeconomic factors. Paradoxically, these patients demonstrate improved survival. Further study is warranted to characterize disease biology in this population.

\section{Acknowledgments}

Funding: None.

\section{Footnote}

Reporting Checklist: The authors have completed the STROBE reporting checklist. Available at https://dx.doi. org/10.21037/jgo-21-207

Peer Review File: Available at https://dx.doi.org/10.21037/ jgo-21-207

Conflicts of Interest: All authors have completed the ICMJE uniform disclosure form (available at https://dx.doi. org/10.21037/jgo-21-207). The authors have no conflicts of interest to declare.

Ethical Statement: The authors are accountable for all aspects of the work in ensuring that questions related to the accuracy or integrity of any part of the work are appropriately investigated and resolved. The study was conducted in accordance with the Declaration of Helsinki (as revised in 2013), and was determined to be exempt from Institutional Review Board (IRB) oversight due to the deidentified nature of the data.

Open Access Statement: This is an Open Access article distributed in accordance with the Creative Commons Attribution-NonCommercial-NoDerivs 4.0 International License (CC BY-NC-ND 4.0), which permits the noncommercial replication and distribution of the article with the strict proviso that no changes or edits are made and the 
original work is properly cited (including links to both the formal publication through the relevant DOI and the license). See: https://creativecommons.org/licenses/by-nc-nd/4.0/.

\section{References}

1. Siegel RL, Miller KD, Jemal A. Cancer statistics, 2019. CA Cancer J Clin 2019;69:7-34.

2. Rawla P, Barsouk A. Epidemiology of gastric cancer: global trends, risk factors and prevention. Prz Gastroenterol 2019;14:26-38.

3. Strong VE, Song KY, Park CH, et al. Comparison of gastric cancer survival following R0 resection in the United States and Korea using an internationally validated nomogram. Ann Surg 2010;251:640-6.

4. Sasako M, Sakuramoto S, Katai H, et al. Five-year outcomes of a randomized phase III trial comparing adjuvant chemotherapy with S-1 versus surgery alone in stage II or III gastric cancer. J Clin Oncol 2011;29:4387-93.

5. Macdonald JS, Smalley SR, Benedetti J, et al. Chemoradiotherapy after surgery compared with surgery alone for adenocarcinoma of the stomach or gastroesophageal junction. N Engl J Med 2001;345:725-30.

6. Noguchi Y, Yoshikawa T, Tsuburaya A, et al. Is gastric carcinoma different between Japan and the United States? Cancer 2000;89:2237-46.

7. Kim GH, Bang SJ, Ende AR, et al. Is screening and surveillance for early detection of gastric cancer needed in Korean Americans? Korean J Intern Med 2015;30:747-58.

8. Yoon H, Kim N, Lee HS, et al. Effect of endoscopic screening at 1-year intervals on the clinicopathologic characteristics and treatment of gastric cancer in South Korea. J Gastroenterol Hepatol 2012;27:928-34.

9. Park CH, Kim EH, Chung H, et al. The optimal endoscopic screening interval for detecting early gastric neoplasms. Gastrointest Endosc 2014;80:253-9.

10. Cunningham D, Allum WH, Stenning SP, et al. Perioperative chemotherapy versus surgery alone for resectable gastroesophageal cancer. N Engl J Med 2006;355:11-20.

11. Songun I, Putter H, Kranenbarg EM, et al. Surgical treatment of gastric cancer: 15-year follow-up results of the randomised nationwide Dutch D1D2 trial. Lancet Oncol 2010;11:439-49.

12. Bickenbach K, Strong VE. Comparisons of Gastric Cancer Treatments: East vs. West. J Gastric Cancer 2012;12:55-62.

13. Mocellin S, McCulloch P, Kazi H, et al. Extent of lymph node dissection for adenocarcinoma of the stomach.
Cochrane Database Syst Rev 2015;(8):CD001964.

14. Theuer CP, Kurosaki T, Ziogas A, et al. Asian patients with gastric carcinoma in the United States exhibit unique clinical features and superior overall and cancer specific survival rates. Cancer 2000;89:1883-92.

15. Wang J, Sun Y, Bertagnolli MM. Comparison of gastric cancer survival between Caucasian and Asian patients treated in the United States: results from the Surveillance Epidemiology and End Results (SEER) database. Ann Surg Oncol 2015;22:2965-71.

16. Kim J, Sun CL, Mailey B, et al. Race and ethnicity correlate with survival in patients with gastric adenocarcinoma. Ann Oncol 2010;21:152-60.

17. Merchant SJ, Kim J, Choi AH, et al. A rising trend in the incidence of advanced gastric cancer in young Hispanic men. Gastric Cancer 2017;20:226-34.

18. Fléjou JF. WHO Classification of digestive tumors: the fourth edition. Ann Pathol 2011;31:S27-31.

19. National Comprehensive Cancer Network. Gastric Cancer (Version 2.2021). Available online: https://www.nccn. org/professionals/physician_gls/pdf/gastric.pdf. Accessed March 31, 2021.

20. Zhang Z, Kim HJ, Lonjon G, et al. Balance diagnostics after propensity score matching. Ann Transl Med 2019;7:16.

21. Lauwers GY, Shimizu M, Correa P, et al. Evaluation of gastric biopsies for neoplasia: differences between Japanese and Western pathologists. Am J Surg Pathol 1999;23:511-8.

22. Schlemper RJ, Itabashi M, Kato Y, et al. Differences in diagnostic criteria for gastric carcinoma between Japanese and western pathologists. Lancet 1997;349:1725-9.

23. Ohtsu A, Yoshida S, Saijo N. Disparities in gastric cancer chemotherapy between the East and West. J Clin Oncol 2006;24:2188-96.

24. Hamashima C; Systematic Review Group and Guideline Development Group for Gastric Cancer Screening Guidelines. Update version of the Japanese Guidelines for Gastric Cancer Screening. Jpn J Clin Oncol 2018;48:673-83.

25. Hirota WK, Zuckerman MJ, Adler DG, et al. ASGE guideline: the role of endoscopy in the surveillance of premalignant conditions of the upper GI tract. Gastrointest Endosc 2006;63:570-80.

26. Gupta S, Li D, El Serag HB, et al. AGA Clinical Practice Guidelines on Management of Gastric Intestinal Metaplasia. Gastroenterology 2020;158:693-702.

27. Al-Batran SE, Hofheinz RD, Pauligk C, et al. Histopathological regression after neoadjuvant docetaxel, oxaliplatin, fluorouracil, and leucovorin versus epirubicin, 
cisplatin, and fluorouracil or capecitabine in patients with resectable gastric or gastro-oesophageal junction adenocarcinoma (FLOT4-AIO): results from the phase 2 part of a multicentre, open-label, randomised phase $2 / 3$ trial. Lancet Oncol 2016;17:1697-708.

28. Wang K, Ren Y, Ma Z, et al. Docetaxel, oxaliplatin, leucovorin, and 5-fluorouracil (FLOT) as preoperative and postoperative chemotherapy compared with surgery followed by chemotherapy for patients with locally advanced gastric cancer: a propensity score-based analysis. Cancer Manag Res 2019;11:3009-20.

29. Al-Batran SE, Homann N, Pauligk C, et al. Perioperative chemotherapy with fluorouracil plus leucovorin, oxaliplatin, and docetaxel versus fluorouracil or capecitabine plus cisplatin and epirubicin for locally advanced, resectable gastric or gastro-oesophageal junction adenocarcinoma (FLOT4): a randomised, phase 2/3 trial. Lancet 2019;393:1948-57.

30. Piessen G, Messager M, Leteurtre E, et al. Signet ring cell histology is an independent predictor of poor prognosis in gastric adenocarcinoma regardless of tumoral clinical presentation. Ann Surg 2009;250:878-87.

31. Bonequi P, Meneses-González F, Correa P, et al. Risk factors for gastric cancer in Latin America: a meta-analysis. Cancer Causes Control 2013;24:217-31.

32. Calderillo-Ruiz G, Takahashi A, Herrera M, et al. Gastric cancer in young Latin women: bad prognostic factors and outcomes. Ann Oncol 2019;30:iv39.

33. Schwarz RE, Zagala-Nevarez K. Ethnic survival differences after gastrectomy for gastric cancer are better explained by factors specific for disease location and individual patient comorbidity. Eur J Surg Oncol 2002;28:214-9.

34. Yao JC, Tseng JF, Worah S, et al. Clinicopathologic behavior of gastric adenocarcinoma in Hispanic patients: analysis of a single institution's experience over 15 years. J Clin Oncol 2005;23:3094-103.

35. Kim DY, Joo JK, Ryu SY, et al. Clinicopathological characteristics and prognosis of carcinoma of the gastric cardia. Dig Surg 2006;23:313-8.

36. Talamonti MS, Kim SP, Yao KA, et al. Surgical outcomes of patients with gastric carcinoma: the importance of primary tumor location and microvessel invasion. Surgery 2003;134:720-7; discussion 727-9.

37. Navaneethan U, Beg MS, Komrokji R, et al. Characteristics of proximal versus distal gastric cancer at the VA: Is there a difference? J Clin Oncol 2008;26:15517.

38. Wang X, Liu F, Li Y, et al. Comparison on Clinicopathological Features, Treatments and Prognosis between Proximal Gastric Cancer and Distal Gastric Cancer: A National Cancer Data Base Analysis. J Cancer 2019;10:3145-53.

39. Claassen YHM, van Amelsfoort RM, Hartgrink HH, et al. Effect of Hospital Volume With Respect to Performing Gastric Cancer Resection on Recurrence and Survival: Results From the CRITICS Trial. Ann Surg 2019;270:1096-102.

40. Smyth EC, Capanu M, Janjigian YY, et al. Tobacco use is associated with increased recurrence and death from gastric cancer. Ann Surg Oncol 2012;19:2088-94.
Cite this article as: Tseng J, Miller JP, Johnson J, Waters K, Gangi A, Gong J, Burch M. Disparities and survival in newly diagnosed gastric cancer in Hispanic patients in the United States: a propensity score matched analysis. J Gastrointest Oncol 2021;12(4):1308-1325. doi: 10.21037/jgo-21-207 